\title{
MicroRNA-150 Regulates Lipid Metabolism and Inflammatory Response
}

Nanlan Luo ${ }^{1}$, W. Timothy Garvey ${ }^{1,2}$, Da-Zhi Wang ${ }^{3}$ and Yuchang Fu ${ }^{1 *}$

${ }^{1}$ Department of Nutrition Sciences, University of Alabama at Birmingham, Birmingham, AL 35294-3360, USA

${ }^{2}$ Birmingham VA Medical Center, Birmingham, AL 35233, USA

${ }^{3}$ Department of Cardiology, Boston Children's Hospital, Harvard Medical School, Boston, MA 02115, USA

\begin{abstract}
Background: MicroRNAs (miRNAs) have emerged as an important class of small molecules that regulate a spectrum of biological processes. However, their roles in the regulation of lipid metabolism and inflammatory response in metabolic syndrome are not completely known. To identify miRNAs and investigate how they are involved in lipid metabolism and inflammatory response in cells and animals and define the function and regulatory mechanism of these microRNAs.
\end{abstract}

Methods and results: We stimulated human THP-1 macrophages with oxLDL and found that one of the miRNAs, miR-150, strongly responded to the lipid accumulation and inflammatory response in these cells. Overexpression of miR-150 in macrophage cells resulted in an increase in lipid accumulation, accompanying with a high expression of several pro-inflammatory cytokines. Conversely, when miR-150 knockout mice were challenged with a high fat diet, these mice presented reduced whole body weight with less fat accumulation, improved systemic glucose tolerance and insulin sensitivity. The expression of pro-inflammatory cytokines in the insulin target adipose tissues was reduced in miR-150 null mice. We identified Adiponectin receptor 2 (AdipoR2) as a potential miR-150 target gene and suggested it may play an important role in miR-150-mediated lipid metabolism and inflammatory response.

Conclusions: These results uncovered novel functions for miR-150 in modulating lipid metabolism and inflammatory response by regulating genes linked to lipid accumulation and related inflammation and provided a firm mechanistic explanation with characterization and determination of critical miR-150 for its associations with the metabolic diseases. These studies will highly impact and benefit metabolic disease research both in vitro and in vivo.

Keywords: MiR-150; Macrophage foam cells; Lipid metabolism; Inflammation

\section{Introduction}

Metabolic syndrome is a powerful risk factor not only for the future development of type 2 diabetes but also cardiovascular disease [1-3]. Alterations of lipid metabolism and inflammatory response in macrophage cells represent a complex pathophysiological process that plays a crucial role in development of metabolic syndrome because macrophage cells accumulate large amounts of lipid and convert to foam cells, which both initiate and actively participate in atherosclerotic lesion development and insulin resistance, as well as other metabolic disorders [4]. Thus, the transformation of macrophages into foam cells is a critical component in the pathophysiological process. A well characterized model system to study the transformation of macrophages to foam cells is the THP-1 human monocytic cell line [5]. THP-1 monocytic cells can be induced to differentiate into macrophages by administration of Phorbol Myristate Acetate (PMA), and resulting macrophages can then be converted to foam cells following treatment with oxidized LowDensity Lipoprotein (oxLDL). The molecular determinants responsible for the transformation of macrophages to foam cells have not been fully elucidated. Here, we decided to capitalize on the advantages of this model system to identify microRNA molecules that are differentially expressed during this cell transformation [6,7]. Toward this end, we performed a microRNA qPCR array utilizing microRNAs isolated from oxLDL-treated and control THP-1 macrophages. One of the microRNA genes which we defined in this screen as being significantly up-regulated in oxLDL-treated macrophage foam cells is microRNA-150 (miR-150).

MiR-150 has been reported mainly expressing in the lymph nodes and spleen and is highly upregulated during the development of mature $\mathrm{T}$ and $\mathrm{B}$ cells; over expression of miR-150 in hematopoietic stem cells had little effect on the formation of either mature T cells or granulocytes or macrophages, but the formation of mature B cells was greatly impaired [8]. Studies have found that opposite expression pattern of miR-150 and c-Myb, a transcription factor controlling multiple steps of lymphocyte development, was detected during B cell differentiation and miR-150 could inhibit c-Myb gene expression and function in vitro $[9,10]$. Furthermore, studies have also shown that deletion of miR-150 in mice caused increased c-Myb expression and B1 cell expansion, conversely, overexpression of miR-150 in mice would reduce c-Myb expression levels and B1 cell population in vivo [11]. These results from both loss- and gain-of-function of miR-150 clearly indicate that miR150 is responsible for $\mathrm{B} 1$ cell differentiation in vivo through $\mathrm{c}-\mathrm{Myb}$ regulation [12].

Recent studies have shown that in human blood cells and cultured THP- 1 cells, miR-150 is selectively packaged into microvesicles (MVs) and actively secreted into the blood or the culture medium; and these THP-1-derived MVs can enter and deliver miR-150 into Human Microvascular Endothelial Cells (HMEC-1), effectively reducing c-Myb expression and enhancing cell migration in HMEC-1 cells [13] Moreover, these studies have found that MVs isolated from the plasma of patients with atherosclerosis contained higher levels of miR-150, and they more effectively promoted HMEC-1 cell migration by blood circulation than MVs from healthy donors.

Our present studies demonstrate for the first time that miR-150 can physiologically modulate metabolic activities and inflammatory

*Corresponding author: Yuchang Fu, Department of Nutrition Sciences, The University of Alabama at Birmingham, Shelby Building/1213, 1825 University Boulevard, Birmingham, AL 35294-0012, USA, Tel: 205-996-4013; Fax: 205-9965896; E-mail: yfu@uab.edu

Received October 23, 2013; Accepted November 15, 2013; Published November 22, 2013

Citation: Luo N, Garvey WT, Wang D-Z, Fu Y (2013) MicroRNA-150 Regulates Lipid Metabolism and Inflammatory Response. J Metabolic Synd 2: 131. doi:10.4172/2167-0943.1000131

Copyright: ( 2013 Luoa N, et al. This is an open-access article distributed under the terms of the Creative Commons Attribution License, which permits unrestricted use, distribution, and reproduction in any medium, provided the original author and source are credited. 
response both in cells and animals by regulating lipid metabolism and inflammatory response. These current studies have revealed a new regulatory role of miR-150 which is related to the mechanism for lipid metabolism and inflammation.

\section{Methods}

\section{Human THP-1 cell culture}

Human monocytic leukemia THP-1 cells were purchased from the American Type Culture Collection (ATCC, Manassas, VA). These cells were cultured in RPMI 1640 medium supplemented with $10 \%$ fetal calf serum (Tissue Culture Biologicals, Tulare, CA), penicillin (100 U/ml) and streptomycin $(100 \mathrm{mg} / \mathrm{ml})$ at $37^{\circ} \mathrm{C}$ in $5 \% \mathrm{CO}_{2}$. THP-1 monocytes were treated with $100 \mathrm{~nm}$ PMA for $24 \mathrm{hr}$ to facilitate differentiation into macrophages. After treatment, the adherent macrophages were washed three times with Phosphate-Buffered Saline (PBS) and incubated with cell culture medium for $24 \mathrm{hr}$ at $37^{\circ} \mathrm{C}$ until addition of $100 \mu \mathrm{g} / \mathrm{mL}$ oxLDL which was purchased from Kalen Biomedical (Montgomery Village, MD).

\section{MicroRNA qPCR array}

Human THP-1 macrophage foam cells (treated with $100 \mu \mathrm{g} / \mathrm{ml}$ of oxLDL for $24 \mathrm{hr}$ ) were harvested for microRNA isolation using a complete miRNA qPCR Array System from Qiagen (Austin, TX). Three RT2 miRNA PCR Arrays were performed and analyzed according to the manufacturer's protocols and software. The selected miRNA molecules were confirmed in oxLDL treated THP-1 macrophage foam cells and the control cells using the real-time PCR methods.

\section{Determination of cholesterol and triglyceride concentrations in THP-1 macrophage foam cells}

The concentrations of cholesterol and triglyceride in THP-1 macrophages foam cells were determined using enzymatic colorimetric assays (Wako, Richmond, VA) according to the manufacturer's protocols. The concentrations of cellular proteins from these macrophage cells were measured with a protein assay kit from Bio-Rad (Hercules, CA).

\section{Overexpression and knockdown of miR-150 in THP-1 cells}

GFP hsa-mir-150 microRNA and inhibitory hsa-mir-150 microRNA lentiviruses $\left(10^{6} \mathrm{pfu} / \mathrm{ml}\right)$ were purchased from the Applied Biological Materials (ABM) (Richmond, BC, Canada). These LentimiRa-GFPhsa-mir-150 and LentimiRa-Off-hsa-miR-150 lentivirus were used to infect $2 \times 10^{5} \mathrm{THP}-1$ cells/well for $24 \mathrm{hr}$ according to the manufacturer's protocols with $53 \%$ to $62 \%$ of efficiency for the infection of cells. These infected THP-1 cells were then treated with $100 \mathrm{~nm}$ PMA for $24 \mathrm{~h}$ to differentiate into macrophages. Then, these macrophages were treated with $100 \mu \mathrm{g} / \mathrm{mL}$ oxLDL for $24 \mathrm{hr}$ to transform them into macrophage foam cells for using in the experiments.

\section{Experimental animals}

Wild type and whole body miR-150 knockout mice were purchased from the Jackson Laboratory for the experiments. To investigate the metabolism of these mice, a chow diet or a high fat diet $(60 \% \mathrm{kcal} \%$ fat) from the Research Diets Company (New Brunswick, NJ) was fed to these mice from 4 weeks to 20 weeks for measuring mouse body weight and analyzing lipid accumulation and gene expression in adipose tissues.

All of these animals were housed in a specific pathogen-free facility with 12-hours light/dark cycles and received a standard laboratory chow diet except for the high fat diet experiments. Only male mice were used for the experiments. All animal procedures were approved by the Institutional Animal Care and Use Committee (IACUC) of the Animal Resources Program (ARP) at the University of Alabama at Birmingham.

\section{Mouse glucose and insulin tolerance testing}

Glucose Tolerance Testing (GTT) and Insulin Tolerance Testing (ITT) in miR-150 knockout and control mice were performed as described previously [14]. Mice were injected with glucose or insulin at 20 or 22 weeks of age after consuming the high fat diet for 16 or 18 weeks. To determine glucose tolerance, animals were first fasted overnight and then given an intraperitoneal injection of glucose solution with $1.0 \mathrm{ml} /$ $\mathrm{kg}$ (1M glucose solution) and glucose concentration was determined in mouse tail blood collected at baseline (prior to injection), and at 30, 60, 90 and 120 minutes post-injection using a HemoCue glucose 201 glucometer (HemoCue USA). To determine insulin tolerance, mice were fasted for 6 hours in the morning of the measurement and then administered an intraperitoneal injection of insulin solution ( $0.5 \mathrm{U}$ insulin/kg body weight). Glucose levels were measured in blood samples collected as above described for the glucose tolerance testing.

\section{Gene expression in THP-1 macrophage foam cells and mouse adipose tissues}

To determine the expression levels of genes coding for lipid metabolism and inflammatory cytokines, total RNA was extracted from THP-1 foam cells or wild type and transgenic mouse adipose tissues using a commercially available TRIzol reagent from Invitrogen (Carlsbad, CA) according to the manufacturer's instructions. The quantitative real-time PCR analysis was using an ABI StepOnePlus Real-Time PCR System. Reactions were carried out in triplicate in a total volume of $20 \mu \mathrm{l}$ using and a SYBR Green QPCR Master Mix (Applied Biosystems). Quantification was calculated using the starting quantity of the cDNA of interest relative to that of $18 \mathrm{~S}$ ribosomal cDNA in the same sample.

\section{Statistics}

Experimental results are reported as the mean \pm SEM. Statistical analyses were conducted using the unpaired Students's $t$-test assuming unequal variance unless otherwise indicated. Significance was defined as the $\mathrm{p}<0.05$.

\section{Results}

\section{MiR-150 is highly induced by oxLDL in macrophage foam} cells

To identify miRNAs and investigate their functional roles in lipid accumulation and inflammatory response in macrophage cells, we treated human THP-1 macrophages with $100 \mu \mathrm{g} / \mathrm{ml}$ of oxLDL to transform these cells into macrophage foam cells with accumulated lipid content inside of these cells. We performed miRNA PCR arrays to identify miRNA molecules that are induced by the lipid accumulation and inflammatory stimulation. Our experimental results showed that miR-150 (average over 4-fold levels, $\mathrm{p}<0.01$ ) was one of the miRNAs which were significantly induced by oxLDL treatment in macrophage foam cells (Figure 1). Increased miR-150 expression was further confirmed with real-time PCR (data not shown).

\section{Overexpression and knockdown of miR-150 in THP-1 cells}

To study biological functions of miR-150, we over express or knockdown miR-150 in THP-1 macrophage cells using lenti viruses. We verified the over expression or knockdown of miR-150 by quantitative RT-PCR (Figure 2A). We found that lipid accumulation 


\section{miR-150 expression}

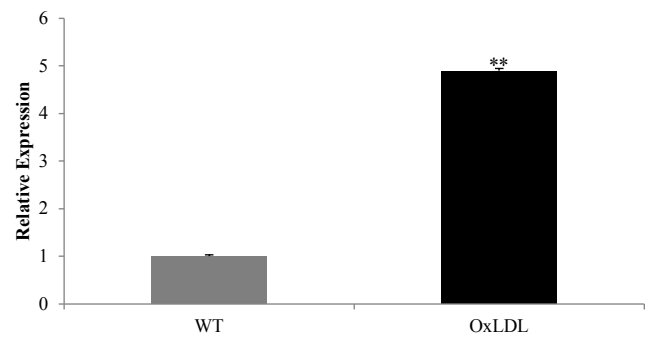

Figure 1: miR-150 is highly induced by oxLDL in THP-1 macrophage foam cells.

Human monocytic leukemia THP-1 cells were treated with $100 \mathrm{nM}$ PMA for $24 \mathrm{~h}$ to facilitate differentiation into macrophages and the macrophages were then treated with $100 \mu \mathrm{g} / \mathrm{mL}$ oxLDL for $24 \mathrm{~h}$ to transform into foam cells. MicroRNAs were isolated by using an RT2 miRNA First Strand Kit (Qiagen) from control THP-1 macrophages (WT) and THP-1 macrophage foam cells (OxLDL) to convert to cDNAs and three RT2 miRNA PCR arrays were performed and analyzed using the complete miRNAqPCR Array System from Qiagen. The expression of miR-150 represented as three separate experiments. Error bars represented the \pm SEM, ${ }^{* *}<<0.01$.

A
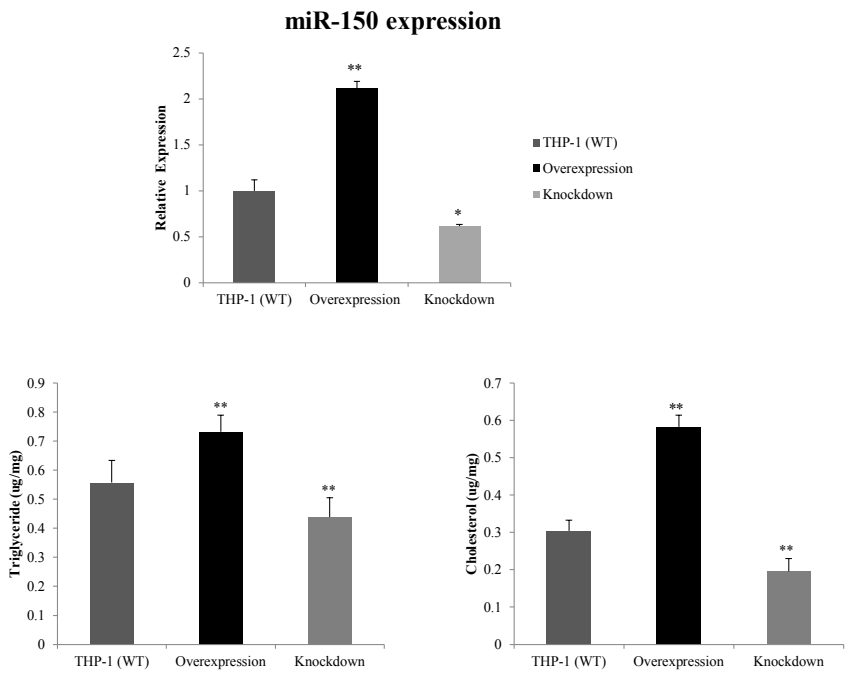

C

Lipid metabolism

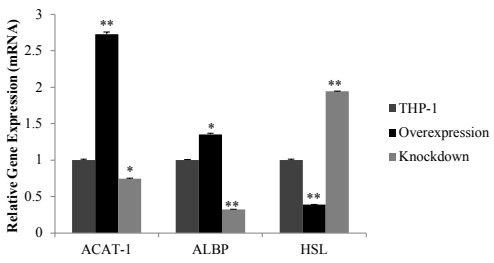

D

Inflammatory response

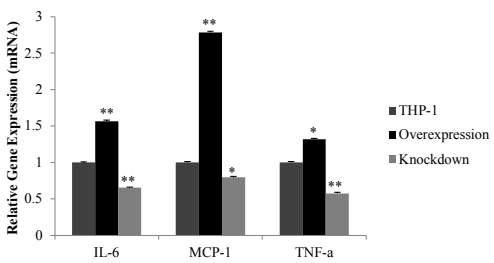

Figure 2: (A) Overexpression and knockdown of miR-150 gene in macrophage cells

LentimiRa-GFP-hsa-mir-150 and LentimiRa-Off-hsa-miR-150 lentiviruues were used to infect $2 \times 10^{5} \mathrm{THP}-1$ cells/well for $24 \mathrm{~h}$ and treated with $100 \mathrm{nM}$ PMA for another $24 \mathrm{~h}$. These macrophages were transformed into foam cells by adding $100 \mu \mathrm{g} / \mathrm{mL}$ oxLDL for $24 \mathrm{~h}$. (A) Expression of miR-150 gene were examined by QPCR analyses in control THP-1 macrophage foam cells (WT), overexpression and knockdown of miR-150 macrophage foam cells. Error bars represented the \pm SEM, ${ }^{*}<<0.05$, ${ }^{* *} p<0.01$. (B) Determination of triglyceride and cholesterol concentrations in macrophage foam cells

The concentrations of triglyceride and cholesterol in THP-1 (WT), overexpression and knockdown of miR-150 macrophages foam cells were determined using enzymatic colorimetric assays. The concentrations of cellular proteins from these macrophage foam cells were measured with protein assay kits. Error bars represented the \pm SEM, ${ }^{* *} \mathrm{p}<0.01$

(C) and (D) Gene expression related to lipid metabolism and inflammatory response in macrophage foam cells

Total RNA was isolated from control THP-1, overexpression and knockdown of miR-150 macrophages foam cells, cDNAs were synthesized, and QPCR was performed with ACAT-1, ALBP, and HSL gene primers for lipid metabolism; IL-6, MCP-1, and TNF- $\mu$ for inflammatory response. Mean \pm SEM from three separate experiments with triplicate samples were presented, ${ }^{*} p<0.05$ and ${ }^{* *} p<0.01$. 
A
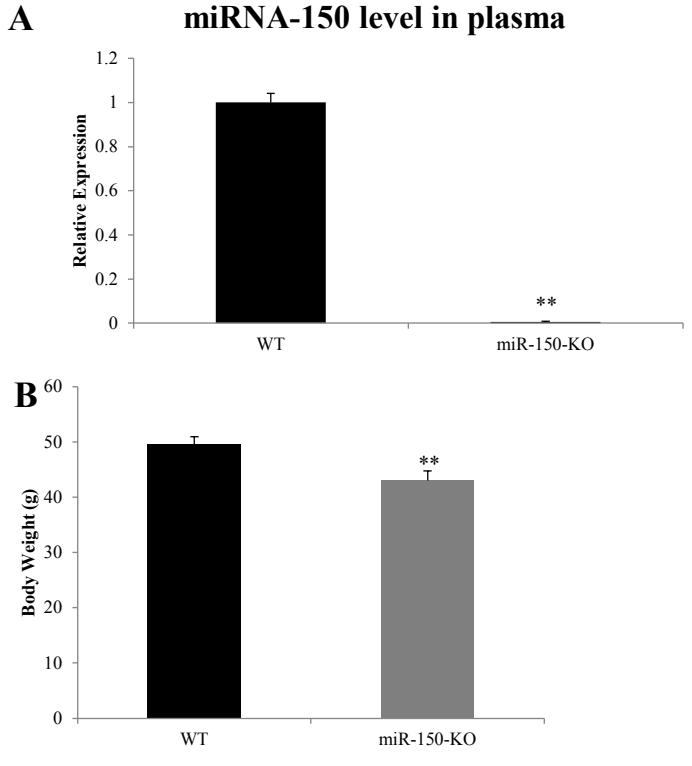

C

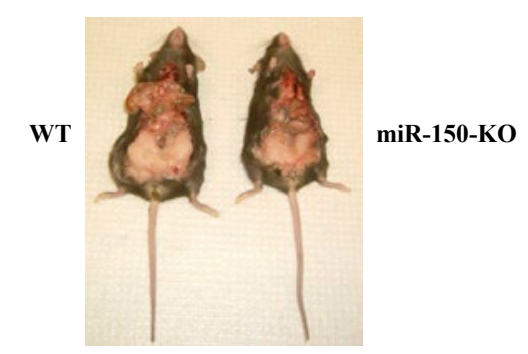

Figure 3: Analysis of miR-150 knockout (miR-150-KO) mice

(A) Measurement of miR-150 levels in mouse plasma

Plasma miR-150 were isolated and collected from plasmas of control wild type (WT) and miR-150 knockout (miR-150-KO) mice at age of 20 weeks using a miRNeasy Serum/Plasma Kit (Qiagen) with a C. elegans miR-39 miRNA mimic as the spike-in control. The expression of miR-150 was examined with QPCR analysis. Each group of eight mice $(n=8)$ was used in the analysis. Error bars represented the \pm SEM, ${ }^{* *} p<0.01$.

(B)Weights of whole body in mice fed with high fat diet

The weights of mouse bodies of control wild type (WT) and miR-150 knockout (miR-150-KO) mice fed a high fat diet for 16 weeks and aged at 20 weeks. ${ }^{* *} p<0.01$ for all of the examined mice and $n=8$ for each mouse group.

(C) Reduced abdominal adipose mass in miR-150 knockout mice

Wild type and miR-150-KO mice at age of 20 weeks under high fat die for 16 weeks were dissected and examined for abdominal fat pads in the miR$150-\mathrm{KO}$ mice compared to control animals. Results represented one of three separate experiments.

(triglyceride and cholesterol) was significantly increased in the miR150 overexpressed macrophage foam cells. In contrast, in the miR-150 knockdown cells, the lipid accumulation was significantly decreased when compared to control THP-1 macrophage foam cells (Figure 2B). We further examined the expression of several key genes related to lipid metabolism, such as, acyl-coenzyme A: Cholesterol acyl transferase 1 (ACAT-1), Adipocyte Lipid-Binding Protein (ALBP/aP2/FABP4) and Hormone Sensitive Lipase (HSL), and to inflammation, including Interleukin 6 (IL-6), Monocyte Chemoatractant Protein 1 (MCP-1) and Tumor Necrosis Factor alpha (TNF- $\alpha$ ) in miR-150 overexpressed or knockdown cells. Our data demonstrated that miR-150 strongly induced the expression of pro-inflammatory cytokines and increased the expression of lipid accumulation genes in the THP-1 macrophage foam cells (Figures 2C and 2D).

\section{MiR-150 knockout mice display reduction of whole body and} fat mass

The above observations prompted us to investigate the in vivo function of miR-150 in lipid metabolism and inflammatory response. MiR-150 has been implicated in the regulation of $\mathrm{B}$ and $\mathrm{T}$ cell development and function and miR-150 knockout mice have been reported viable and fertile [11]. We first examined the levels of miR-150 in plasma of wild type and miR-150 knockout mice and we confirmed the absence of miR-150 expression in knockout mice (Figure 3A). Next, we assessed whether growth and development were affected in miR-150 knockout mice compared to wild type mice. There were no significant differences in reproduction, food consumption, or development between control and knockout mice when these animals were fed normal chow or the high fat diet (data not shown). However, compared to wild type mice, miR-150 knockout mice had significantly lower body weight (average 12\%) when these mice fed with a high fat diet $(60 \%$ $\mathrm{kcal} \%$ fat) for 16 weeks at age of 20 weeks (Figure 3B).

To confirm these data, we dissected wild type and miR-150 knockout mice and found significantly reduced abdominal adipose masses in knockout mice when compared to control wild type animals (Figure 3C). Our results suggest that deficiency of miR-150 in mice can significantly affect adiposity, especially adipose tissue accumulation under the high fat diet condition.

\section{Decreased lipid accumulation and pro-inflammatory gene} expression in miR-150 knockout mice

To determine the function of miR-150 on lipid metabolism and inflammatory response, mouse adipose tissues were isolated from
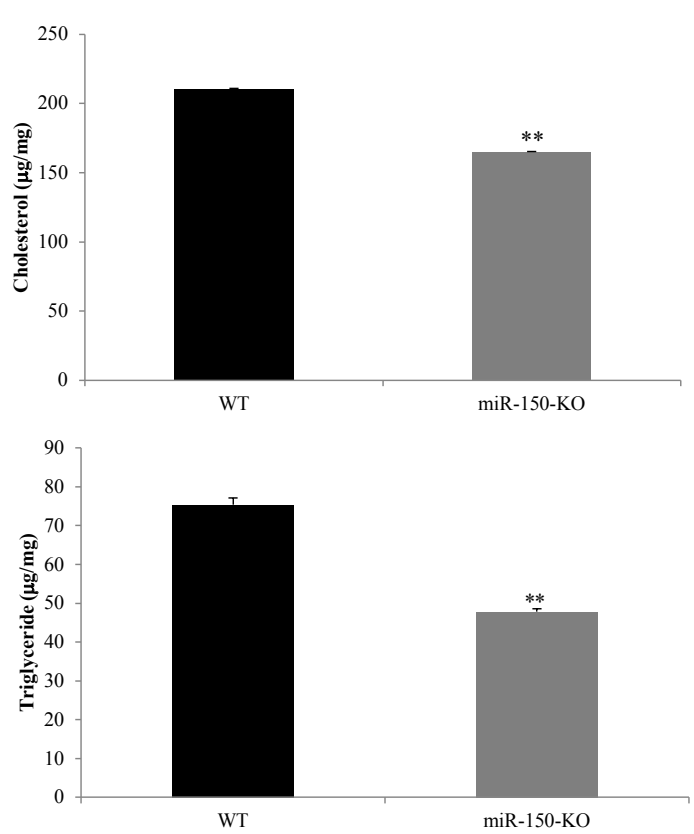

Figure 4: Changes of lipid accumulation in mouse adipose tissues Mouse abdominal fat tissue masses from 20-week-old control wild type (WT) and miR-150 knockout (miR-150-KO) mice fed the high fat diet for 16 weeks were isolated. Cellular accumulation of cholesterol and triglyceride was assessed in the WT and miR-150-KO adipose tissues using enzymatic colorimetric kits from Wako Company. Cellular lipid mass was normalized using cellular protein levels which were determined using a protein analysis kit from the Bio-Rad. Mean \pm SEM from three separate experiments with triplicate samples ( $n=9$ for each group) were examined, ${ }^{* *} p<0.01$. 
20-week-old control (WT) and miR-150 knockout (miR-150-KO) mice and the cellular contents of cholesterol and triglyceride were determined. Both cholesterol (maximal average 16\%) and triglyceride (maximal average 33\%) levels in adipose tissues were significantly reduced $(p<0.01)$ in miR-150 knockout mice under high fat feeding condition for 16 weeks (Figures $4 \mathrm{~A}$ and $4 \mathrm{~B}$ ). We also examined the impact of miR150 gene knockout on lipid metabolism and inflammatory response gene expression in adipose tissues and we found a reduced expression of ALBP, which is for lipid loading and pro-inflammatory cytokines, IL-6, MCP-1 and TNF- $\alpha$, in miR-150 knockout in mice. However, we observed an increased expression of HSL, which is for lipid hydrolyzing and anti-inflammatory cytokine IL-10 in adipose tissues of miR-150 knockout mice (Figure 5A).

To further investigate whether the reduced lipid accumulation in the metabolically active adipose tissues of miR-150 knockout mice could influence the change of macrophage subtype specific markers, we next examined the expression of macrophage-specific F4/80, macrophage galactose $\mathrm{N}$-acetyl-galactosamine specific lectin $1(\mathrm{Mgl}$ 1), a marker for alternatively activated Macrophage (M2) phenotype, and Chemokine Receptor 2 (CCR2), a marker for classically activated macrophage (M1) phenotype, in adipose tissues of miR-150 knockout mice. As expected, the percentage of these pro-inflammatory markers, F4/80 (average 26\%, p <0.05) and CCR2 (average 61\%, p<0.01), were markedly lower in adipose tissues of miR-150 knockout mice (Figure $5 \mathrm{~B})$. In contrast, the anti-inflammatory marker, $\mathrm{Mgl} 1$, was significantly higher (average $82 \%, \mathrm{p}<0.01$ ) in adipose tissues of miR-150-KO mice than those from control wild type mice. Thus, elimination of miR-150
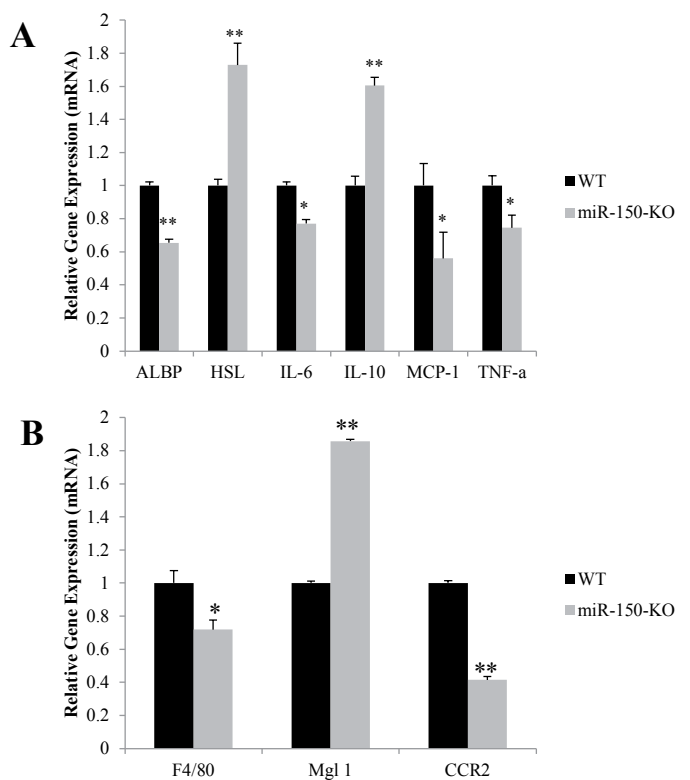

Figure 5: Effects of miR-150 deficiency on gene expression in mouse adipose tissues

Total RNA was isolated from adipose tissues of control wild type (WT) and miR150 knockout (miR-150-KO) mice fed with high fat diet for 16 weeks at age of 20 weeks, cDNAs were synthesized, and QPCR was performed and analyzed. (A) Gene expression linked to lipid metabolism and inflammatory response ALBP and HSL gene primers for lipid metabolism; and IL-6, IL-10, MCP-1, and TNF- $\mu$ for inflammatory response were used in the QPCR experiments.

(B) Changed expression pattern of macrophage-specific markers

Expression of macrophage-specific markers, F4/80, Mgl 1, and CCR2, in adipose tissues from the control (WT) and miR-150 knockout (miR-150-KO) mice were examined by using QPCR analysis. All of experiments are mean \pm SEM from three separate samples with triplicate sets ( $n=12$ for each group) were examined, ${ }^{*} p<0.05$ and ${ }^{* *} p<0.01$.

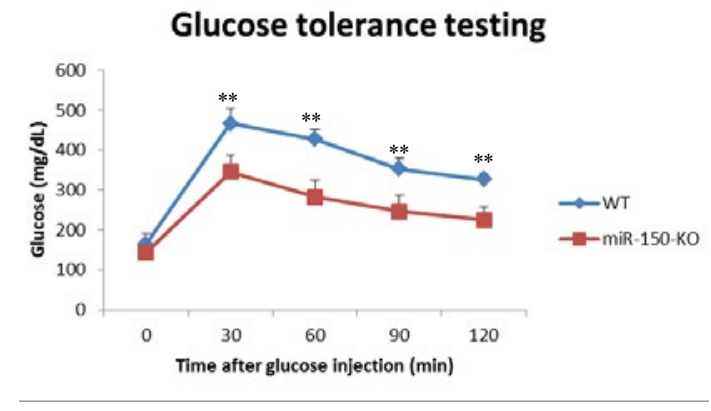

Insulin tolerance testing

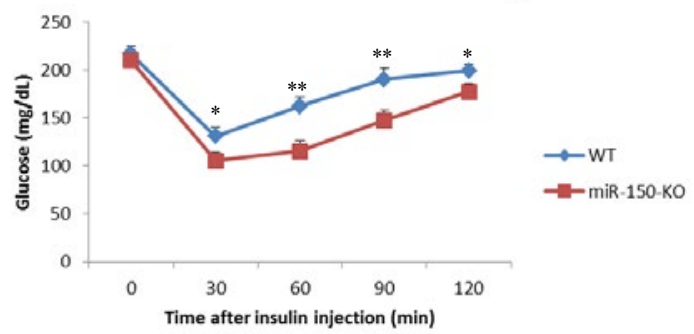

Figure 6: Glucose tolerance tests (GTT) and insulin tolerance tests (ITT) in mice

Glucose tolerance tests were performed on control wild type (WT) and miR150 knockout (miR-150-KO) mice fed with high fat diet for 16 weeks at age of 20 weeks. Experiments were performed in fasting overnight male mice. Glucose solutions were injected into peritoneal cavity at the dose of $1.0 \mathrm{ml} / \mathrm{kg}$ (1M glucose solution). Blood was collected via tail vein at the indicated time points. Glucose concentration in plasma was measured using a glucometer (Precision); $n=10$ in each group of mice. Insulin tolerance tests were performed on control wild type and miR-150 knockout mice under high fat diet condition for 18 weeks at age of 22 weeks. Experiments were conducted similar as the described above for the glucose tolerance tests but fasting 6 hours before the injections and insulin solutions were injected into peritoneal cavity at the dose of $0.5 \mathrm{U} / \mathrm{kg}$ body weight. Blood was collected via tail vein at the indicated time points, and glucose levels were measured; $n=10$ in each group of animals, Error bars represented the \pm SEM, ${ }^{*} p<0.05$ and ${ }^{* *} p<0.01$.

resulted in favorable metabolic macrophage subtype changes in vivo.

Improved glucose tolerance and insulin sensitivity in miR150 knockout mice

To investigate the function of miR-150 in systemic insulin sensitivity in vivo, we performed Glucose Tolerance Tests (GTT) and Insulin Tolerance Tests (ITT) in control and miR-150-KO mice fed with high fat diet for 16 weeks at age of 20 weeks. We found that plasma glucose levels were consistently lower $(p<0.01)$ during the glucose tolerance tests in miR-150-KO mice when compared to that of control wild type animals (Figure 6A). Similarly, the plasma glucose levels were also consistently lower $(p<0.05$ or 0.01$)$ when insulin was injected for the insulin tolerance tests than the levels in miR-150-KO mice (Figure $6 \mathrm{~B})$. We conclude that knockout of miR-150 gene in mice influences systemic metabolism in distal tissues as demonstrated by improved glucose tolerance and insulin sensitivity in these animals.

\section{AdipoR2 is a miR-150 target in mouse adipose tissues}

Finally, we attempted to determine miR-150 targets in adipose tissues. Based on the miRDB database for miRNA target prediction and functional annotations $[15,16]$, AdipoR2 is one of the miR-150 target genes in both human and mouse. We experimentally examined adiponectin receptor 2 (AdipoR2) expression in adipose tissues of both miR-150 knockout and wild type control mice. AdipoR2 expression was significant increased (average $33 \%, \mathrm{p}<0.01$ ) in adipose tissues of 


\section{AdipoR2 expression}

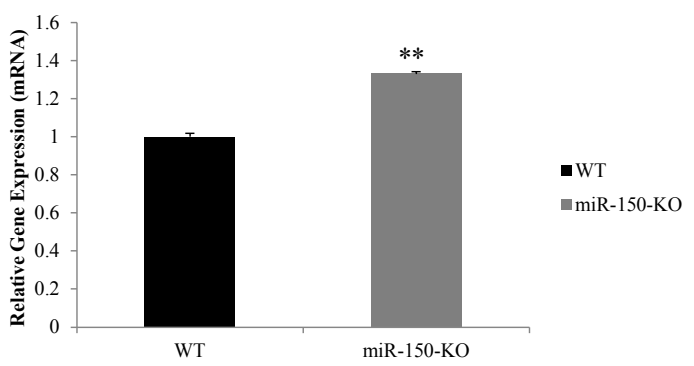

Figure 7: Analysis of adiponectin receptor 2 (AdipoR2) gene expression in mouse adipose tissues

Control wild type (WT) and miR-150 knockout (miR-150-KO) mice were fed with the high fat diet for 18 weeks at age of 22 weeks; abdominal adipose tissues were dissected from these mice and total RNA was isolated from these tissues: cDNAs were synthesized, and QPCR was performed with AdipoR2 gene primers for gene expression. Mean \pm SEM from three separate experiments with triplicate samples ( $n=9$ for each group) were presented, ${ }^{* *} p<0.01$.

miR-150 knockout mice when compared to those in control wild type mice (Figure 7). Thus, the knockout of miR-150 in mice altered the expression of one important adiponectin receptor gene in metabolically active adipose tissues in a manner that would tend to promote lipid metabolism and reduce inflammation in these tissues.

\section{Discussion}

As previously demonstrated, miR-150 was involved in regulating B cell development [8] and megakaryocyte differentiation [17,18]; although the evidence has shown that except of lymphocytes miR150 does not influence on the formation of either mature $\mathrm{T}$ cells or granulocytes or macrophages in hematopoietic stem cells, considering that interactions among these immune cells to respond to pathogens, miR-150 should have a vital role in the immune system [19]. Since lymphoid blood cells tightly are correlated with corresponding white blood cell counts, as one of the cancer biomarkers which have high stability in plasma, association with disease states, and ease of sensitive measurement, miR-150 has been investigated in numerous cancer studies [20,21]. In addition of cancer, disorders of the immune system can also result in autoimmune and inflammatory diseases [22,23].

It is well known that overnutrition or high fat diets are associated with autoimmune and inflammatory diseases such as diabetes and obesity; here, high fat diets exert multiple deleterious effects to key metabolic tissues in vivo, and immune cells, such as macrophages, $\mathrm{T}$ cells, and B cells, have all been implicated to play prominent roles in the inflammatory processes [24]. Recent studies have revealed that miR-150 secretion was significantly increased in ob/ob diabetic mice to promote angiogenesis in these animals [25]. Recent studies have also suggested that microRNAs play an important role in regulating lipid and glucose metabolism [26].

Previous studies have identified two cell-surface trans-membrane receptors, AdipoR1 and AdipoR2, for adiponectin action [27], and the adiponectin action is known to signal through these receptors and the docking protein APPL1 [28]. Simultaneous disruption of both AdipoR1 and AdipoR2 abolished adiponectin binding and actions, resulting in increased triglyceride content, inflammation and oxidative stress in adipose tissue, and thus leading to insulin resistance and marked glucose intolerance [29]. Although recent studies have indicated that microRNAs play an important role in metabolic syndrome [26], currently, the interactions among microRNAs and AdipoR2 are completely undefined yet. Here, we have demonstrated that AdipoR2 was regulated by miR-150 as its one of the potential target genes and the interactions between miR-150 and AdipoR 2 may play a critical role in physiological metabolic activities in cells/tissues; further investigations should reveal more detailed regulatory mechanism of the function of miR-150 involved in metabolism and inflammation.

Our current studies have for the first time identified that miR150 was highly induced by oxLDL stimulation in human THP-1 macrophage foam cells; and its expression and function were related to lipid accumulation in these cells by regulating genes that are involved in lipid metabolism, such as, ACAT-1, ALBP, and HSL. Since the lipid metabolic activity and miR-150 itself are highly related to immune/ inflammatory response in cells, cytokines, including IL-6, IL-10, MCP1 , and TNF- $\alpha$, are also regulated by the expression of miR-150 in these cells. We have further used miR-150 knockout mice to confirm the important roles of miR-150 in regulating these key gene expression related to lipid metabolism, inflammatory response, glucose tolerance and insulin resistance in vivo. Our results have indicated that miR150 was involved in high fat diet induced obesity/diabetes through regulating lipid metabolism and inflammatory response, probably through one of its target genes, AdipoR2, in vivo.

In conclusion, our current studies suggest that miR-150 can physiologically modulate metabolic activities and inflammatory response both in cells and animals by mainly regulating lipid metabolism and inflammatory response. These studies have revealed a new regulatory role of miR-150 and the related mechanism for lipid metabolism and inflammation, suggesting that miR-150 maybe a potential pharmaceutical candidate for prevention and therapy of metabolic disorders in the future.

\section{Acknowledgments}

We thank the UAB Diabetes Research and Training Center for providing outstanding core services (NIH P30 DK-56336). This work was supported by grants from American Diabetes Association (1-13-IN-19) to YF, a grant from NIH (DK083562) to TG and a grant from NIH (HL085635) to DZW.

\section{Reference}

1. Reaven GM (1988) Banting lecture 1988. Role of insulin resistance in human disease. Diabetes 37: 1595-1607.

2. Reaven GM (1991) Insulin resistance, hyperinsulinemia, hypertriglyceridemia and hypertension. Parallels between human disease and rodent models. Diabetes Care 14: 195-202.

3. Nigro J, Osman N, Dart AM, Little PJ (2006) Insulin resistance and atherosclerosis. Endocr Rev 27: 242-259.

4. Steinberg D (1997) Low density lipoprotein oxidation and its pathobiological significance. J Biol Chem 272: 20963-20966.

5. Auwerx J (1991) The human leukemia cell line, THP-1: a multifacetted mode for the study of monocyte-macrophage differentiation. Experientia 47: 22-31.

6. Wang N, Tabas I, Winchester R, Ravalli S, Rabbani LE, et al. (1996) Interleukin 8 is induced by cholesterol loading of macrophages and expressed by macrophage foam cells in human atheroma. J Biol Chem 271: 8837-8842.

7. Jang MK, Choi MS, Park YB (1999) Regulation of ferritin light chain gene expression by oxidized low-density lipoproteins in human monocytic THP-1 cells. Biochem Biophys Res Commun 265: 577-583.

8. Zhou B, Wang S, Mayr C, Bartel DP, Lodish HF (2007) miR-150, a microRNA expressed in mature $B$ and $T$ cells, blocks early $B$ cell development when expressed prematurely. Proc Natl Acad Sci U S A 104: 7080-7085.

9. Monticelli S, Ansel KM, Xiao C, Socci ND, Krichevsky AM, et al. (2005) MicroRNA profiling of the murine hematopoietic system. Genome Biol 6: R71.

10. Thomas MD, Kremer CS, Ravichandran KS, Rajewsky K, Bender TP (2005) c-Myb is critical for B cell development and maintenance of follicular B cells. Immunity 23: $275-286$. 
Citation: Luo N, Garvey WT, Wang D-Z, Fu Y (2013) MicroRNA-150 Regulates Lipid Metabolism and Inflammatory Response. J Metabolic Synd 2: 131. doi:10.4172/2167-0943.1000131

Page 7 of 7

11. Xiao C, Calado DP, Galler G, Thai TH, Patterson HC, et al. (2007) MiR-150 controls $\mathrm{B}$ cell differentiation by targeting the transcription factor c-Myb. Cell 131: 146-159.

12. Park CY, Choi YS, McManus MT (2010) Analysis of microRNA knockouts in mice. Hum Mol Genet 19: R169-175.

13. Zhang Y, Liu D, Chen X, Li J, Li L, et al. (2010) Secreted monocytic miR-150 enhances targeted endothelial cell migration. Mol Cell 39: 133-144.

14. Maeda K, Cao H, Kono K, Gorgun CZ, Furuhashi M, et al. (2005) Adipocyte/ macrophage fatty acid binding proteins control integrated metabolic responses in obesity and diabetes. Cell Metab 1: 107-119.

15. Wang X, EI Naqa IM (2008) Prediction of both conserved and nonconserved microRNA targets in animals. Bioinformatics 24: 325-332.

16. Wang $X(2008)$ miRDB: a microRNA target prediction and functional annotation database with a wiki interface. RNA 14: 1012-1017.

17. Lu J, Guo S, Ebert BL, Zhang H, Peng X, et al. (2008) MicroRNA-mediated control of cell fate in megakaryocyte-erythrocyte progenitors. Dev Cell 14: 843853.

18. Barroga CF, Pham H, Kaushansky K (2008) Thrombopoietin regulates C-Myb expression by modulating micro RNA 150 expression. Exp Hematol 36: 15851592.

19. Tsitsiou E, Lindsay MA (2009) microRNAs and the immune response. Curr Opin Pharmacol 9: 514-520.

20. Garzon R, Croce CM (2008) MicroRNAs in normal and malignant hematopoiesis. Curr Opin Hematol 15: 352-358.
21. Pritchard CC, Kroh E, Wood B, Arroyo JD, Dougherty KJ, et al. (2012) Blood cell origin of circulating microRNAs: a cautionary note for cancer biomarker studies. Cancer Prev Res (Phila) 5: 492-497.

22. Coussens LM, Werb Z (2001) Inflammatory cells and cancer: think different! J Exp Med 193: F23-26.

23. O'Byrne KJ, Dalgleish AG (2001) Chronic immune activation and inflammation as the cause of malignancy. $\mathrm{Br} \mathrm{J}$ Cancer 85: 473-483.

24. Swindell WR, Johnston A, Gudjonsson JE (2010) Transcriptional profiles of leukocyte populations provide a tool for interpreting gene expression patterns associated with high fat diet in mice. PLoS One 5: e11861.

25. Li J, Zhang Y, Liu Y, Dai X, Li W, et al. (2013) Microvesicle-mediated transfer of microRNA-150 from monocytes to endothelial cells promotes angiogenesis. J Biol Chem 288: 23586-23596.

26. Fernández-Hernando C, Ramírez CM, Goedeke L, Suárez Y (2013) MicroRNAs in metabolic disease. Arterioscler Thromb Vasc Biol 33: 178-185.

27. Yamauchi T, Kamon J, Ito Y, Tsuchida A, Yokomizo T, et al. (2003) Cloning of adiponectin receptors that mediate antidiabetic metabolic effects. Nature 423 762-769.

28. Mao X, Kikani CK, Riojas RA, Langlais P, Wang L, et al. (2006) APPL1 binds to adiponectin receptors and mediates adiponectin signalling and function. Nat Cell Biol 8: 516-523.

29. Yamauchi T, Nio Y, Maki T, Kobayashi M, Takazawa T, et al. (2007) Targeted disruption of AdipoR1 and AdipoR2 causes abrogation of adiponectin binding and metabolic actions. Nat Med 13: 332-339. 